\title{
Concepciones sobre el infinito: Un estudio a nivel universitario
}

\begin{tabular}{|c|c|c|}
\hline Javier González & Armando Morales & José María Sigarreta \\
\hline jg_mendieta@hotmail.com & armando280@hotmail.com & josemariasigarretaalamira@hotmail.com \\
\hline Universidad Autónoma de Guerrero & Universidad Autónoma de Guerrero & Universidad Autónoma de Guerrero \\
\hline México & México & México \\
\hline Recibido: 8 Mayo 2012 & Aceptado: $8 \mathrm{~N}$ & nbre 2012 \\
\hline
\end{tabular}

Resumen. En este artículo, se reportan los resultados sobre las concepciones del infinito que prevalecen en los estudiantes de la Licenciatura en Matemáticas de la Universidad Autónoma de Guerrero, México cuando se enfrentan a procesos infinitos. Se hace un estudio explicativo-descriptivo de las diferentes acepciones del infinito más utilizadas en este nivel de Licenciatura en Matemáticas y en particular, como elemento de refuerzo teórico, asumimos que un proceso infinito tiene situación límite si existe alguna variable asociada al proceso, que cumpla el principio de exhausción. En esa dirección, se exponen los elementos metodológicos que sustentan la investigación. Resulta atinado plantear, que aunque en muchos de los casos analizados, prevalece una cierta concepción del infinito actual mal utilizada, se concluye que el infinito que predomina en los estudiantes cuando analizan procesos infinitos, es el infinito potencial.

Palabras clave: Infinito potencial y actual, proceso infinito, situación límite, principio de exhausción.

Abstract. In this article, we report the conceptions of infinity that are present in students of Mathematics at the Autonomous University of Guerrero, México when they work with infinite processes. Furthermore, we show the meanings of the concept of infinity most used in school and in particular as a reinforcing element theory we assume that a process has infinite limit situation if there is any variable associated with the process, which satisfies the principle of exhaustion. In this direction, we present methodological elements that ensure the investigation. Although many of the cases analyzed, there prevails a certain conception of the actual infinite, we conclude that the infinite used in our students is the potential infinite

KeyWords: Potential and actual infinite, infinite process, limit situation, principle of exhaustion

\subsection{Introducción}

Este trabajo es motivado a partir del reporte de investigaciones sobre dificultades asociadas al concepto de límite finito e infinito de Camacho, A. y Aguirre, M. (2001), Engler, A. et al.(2008), Antibi, A.(1996), Reyes, L. E.(2012) y sobre los obstáculos epistemológicos según Cornu (1983), en específico, «Sobre generalización de las propiedades de los procesos finitos a los procesos infinitos» y «Sentido común de la palabra límite, lo que induce a concepciones persistentes como barrera infranqueable o como último término de un proceso». Las ideas anteriores sobre obstáculos, también han sido explicadas en investigaciones como en Díaz, M. (2009), en el que establece que los griegos por mucho tiempo, prescindieron de la situación límite de los procesos infinitos, y en su lugar vieron el método de exhausción, como el ideal y riguroso para la justificación de sus teorías matemáticas.

Por otro lado, Hitt, F. (2003), señala que el infinito es un obstáculo para el aprendizaje del límite, ya que según sus estudios, los alumnos confunden entre procesos infinitos(infinito potencial) y situación límite (culminación del proceso infinito, infinito actual), por ejemplo, para las expresiones $9 / 10+9 / 100+9 / 1000+\ldots$ y 1 , los estudiantes 
no logran explicar que en la situación límite del proceso infinito $9 / 10+9 / 100+9 / 1000+\ldots$ tiende a 1 .

La finalidad del trabajo es analizar situaciones que involucran procesos infinitos, con ello podremos indagar sobre el tipo de infinito que prevalece en los estudiantes cuando analizan este tipo de situaciones. También se considera que éstos análisis y por lo tanto su reporte, tiene mucha importancia para quienes se dedican a la enseñanza del Cálculo y del Análisis, pues estos hallazgos pueden servir como punto de partida para futuras investigaciones que pretenden incidir en la solución del problema de la enseñanza-aprendizaje del Cálculo y del Análisis, y en particular del concepto de límite en el nivel universitario.

\subsection{Infinito potencial e infinito actual: Una descripción general}

Infinito potencial

El infinito potencial emerge del estudio de las sucesiones numéricas, pues en la práctica existen muchas sucesiones que aparecen de forma natural al aplicar algún algoritmo o al resolver problemas que llevan implícito un proceso de aproximación (Moreno y Waldeg,1986). Algunos ejemplos son: 1) El algoritmo de la división, a parte de proporcionar un método para calcular el cociente entre dos enteros cualesquiera, permite generar una sucesión numérica, ya que en cada paso del proceso de la división, se genera una aproximación al cociente de los números. 2) El algoritmo de la raíz cuadrada: en este caso el algoritmo nos da una aproximación por defecto, y en cada paso del proceso, obtenemos términos cada vez más cercanos a la raíz cuadrada del valor indicado, en particular, se genera una sucesión creciente y acotada (lenguaje moderno).

En la geometría también se encuentran situaciones en donde la idea del infinito potencial se manifiesta como un proceso reiterativo, por ejemplo:«Determinar la circunferencia de un círculo de diámetro unidad», este caso, lleva de forma natural a buscar el valor numérico de $\pi$. El problema fue resuelto por el griego Arquímedes (287-212 a. C.), el método que utilizó consistió en inscribir polígonos regulares en el círculo, y determinar en cada inscripción la suma de las longitudes de los lados, se genera una sucesión de perímetros de polígonos regulares de $4,8,16, \ldots, 2^{n+1}, \ldots$ lados, tales que cada polígono tiene sus vértices coincidiendo con algunos de los vértices del polígono siguiente. Para determinar los perímetros de los polígonos, se utilizan conceptos de la geometría plana.

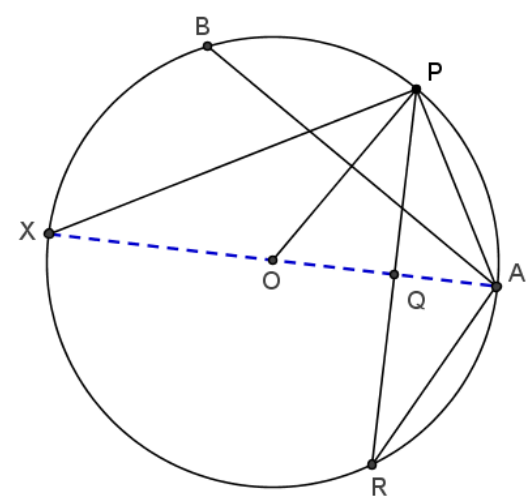

Figura 1.1

En la Figura 1, sea $A O X$ el diámetro de un círculo de radio $r$. Sea $\overline{A B}$ el lado de un polígono regular de $2^{n+1}$ lados, cada uno de longitud $S_{n}$ y sea $\overline{A P}$ el lado de un polígono regular de $2^{(n+1)+1}$ lados de longitud $S_{n+1}$. A partir de razonamientos geométricos, se establece la relación entre $S_{n+1}$ y $S_{n}$. De esta manera, $S_{n+1}=\sqrt{2 r^{2}-r \sqrt{4 r^{2}-\left(S_{n}\right)^{2}}}$. Así, si el círculo tiene diámetro uno, entonces, el lado del cuadrado mide $S_{1}=\frac{1}{2} \sqrt{2}$, el lado del octágono mide $S_{2}=\frac{1}{2} \sqrt{2-\sqrt{2}}$ y así sucesivamente, de esta forma, se genera una sucesión de longitudes de lados y por lo tanto 
una sucesión de perímetros, como se observa en la siguiente tabla:

\begin{tabular}{|l|l|l|l|}
\hline$n$ & Número de lados $2^{n+1}$ & Longitud del $S_{n}$ & Perímetro $P n=2^{n+1} S_{n}$ \\
\hline 1 & 4 & $\frac{1}{2} \sqrt{2}$ & 2.8284 \\
2 & 8 & $\frac{1}{2} \sqrt{2-\sqrt{2}}$ & 3.0615 \\
3 & 16 & $\frac{1}{2} \sqrt{2-\sqrt{2+\sqrt{2}}}$ & 3.1214 \\
4 & 32 & $\frac{1}{2} \sqrt{2-\sqrt{2+\sqrt{2+\sqrt{2}}}}$ & 3.1365 \\
$\vdots$ & $\vdots$ & $\vdots$ & $\vdots$ \\
\hline
\end{tabular}

En la cuarta columna de la tabla anterior, se observan algunos términos de la sucesión de perímetros de los cuatro primeros polígonos inscritos en el círculo de diámetro unidad, cada uno de los términos es un valor aproximado al valor de $\pi$, este ejemplo evidencia que los griegos conocieron del infinito potencial y lo utilizaron para estudiar los procesos infinitos, los cuales validaron haciendo uso del método de axhausción.

Por otra parte, algunos investigadores como De Lorenzo (2001), consideran que el infinito potencial, ha quedado conceptualizado en el número natural, al mencionar que siempre que se propone un número natural dado, es posible proponer uno mayor que el anterior, de tal suerte que no hay un último número natural, pues cada uno de ellos a la vez, tiene un sucesor.

Se han dado algunos ejemplos en donde se identifica el concepto de infinito, en particular el infinito potencial como proceso de reiteración que tiende a culminar, por ejemplo, la superficie del círculo es el límite hacia el cual convergen las superficies de los polígonos inscritos o circunscritos, conforme crece su número de lados; un crecer cada vez más que podría expresarse indicando que el número de lados de los polígonos inscritos o circunscritos tiende a $\infty$. La propia circunferencia puede identificarse, en el límite, con un polígono de infinitos lados infinitesimales.

$\mathrm{Al}$ analizar los ejemplos anteriores, se observa que el infinito ya no aparece como proceso, sino como producto de ese proceso, es decir, una situación límite. En ese sentido, infinito designa ahora el número que completa la sucesión de los números naturales, ese número apunta al límite de valores numéricos sucesivos de una misma variable que crece cada vez más, indicándolo con $+\infty$, que completa junto con $-\infty$, la recta real o del continuo (De Lorenzo, 2001).

Infinito actual

Algunos autores como (De Lorenzo, 2001; Le Goff, 2001) consideran el infinito actual, como un concepto originado en un contexto geométrico, consideran que es un infinito ilimitado, métrico, que permite la cuantificación y la resolución de problemas del mundo real. Viene requerido desde el propio proceso e incluye elementos ideales (número infinito, punto infinito y series con infinitos elementos). El salto del infinito potencial al actual, es donde encuentra sentido el proceso demostrativo de la inducción completa, según Blaise Pascal (citado en De Lorenzo, J., 2001) cuando afirmaba:

Conocemos que hay un infinito e ignoramos su naturaleza. Cómo sabemos que los números son infinitos, que es verdad que hay un infinito numérico.

Como ejemplo de infinito actual se tiene el conjunto de los números naturales, que supone admite un nuevo número cardinal transfinito $\aleph_{0}$. Este infinito actual como conjunto y su número cardinal transfinito asociado proceden del proceso de comparación biyectiva, otro proceso conceptualizador que se une al de la iteración. Aquí se admite que los conjuntos están dados en acto, es decir, no se va construyendo elemento por elemento, tampoco importa que a estos conjuntos, una vez dados en acto, se les pueda aplicar un proceso de iteración. Naturalmente surgen algunas preguntas tales como ¿hay un solo infinito actual? ¿hay muchos? La infinitud aparece ahora como concepto derivado y no primitivo, definible en términos de conjunto y de aplicación biyectiva entre conjuntos. Un conjunto se dirá simplemente infinito si se puede poner en correspondencia biyectiva con una de sus partes propias. Así, el conjunto de los números naturales es biyectivo con el conjunto de los números primos. Lo cual implica que ambos 
conjuntos poseen la misma cifra de números y han de tener el mismo cardinal.

Por tanto, desde lo iterable se puede decir que los números primos aparecen en menor cantidad que los números naturales de los que forman parte y a los que originan, pues según el teorema fundamental de la Aritmética "todo entero mayor o igual que 2, se descompone de manera única, expresándose como el producto de sus factores primos", desde el argumento euclídeo "el todo es mayor que cualquiera de sus partes propias", todas estas situaciones quedan invalidadas cuando se tratan conjuntos dados en acto. Ante la pregunta ¿habrá un sólo tipo de infinito actual, el asociado con el conjunto de los números naturales dados en acto? En términos equivalentes, ¿habrá un único número cardinal transfinito? Traspasada la noción de ilimitación y aceptada la idea de un infinito actual, parece ser más plausible esperar que no hubiera más que un infinito.

Por su parte Cantor demolió tal suposición al demostrar la existencia de toda una escala de transfinitos. Creó incluso el camino, el método de la diagonal, con el que se demuestra que la cardinalidad de $\mathbb{N}$ es menor que la cardinalidad de $\mathbb{R}$ y se puede argumentar que dado cualquier conjunto, habrá siempre un conjunto cuyo cardinal es de infinitud mayor (Hernández, 2003). El punto de partida es que los conjuntos tienen que estar dados, pues de lo contrario no se podrán comparar. Luego, si un conjunto está dado, también lo están sus partes propias, es decir, el conjunto de todos los subconjuntos de dicho conjunto, lo que se denomina su conjunto potencia, cuyo cardinal es mayor que el correspondiente al del conjunto de partida. Y aquí se tiene otro proceso creador de nuevos cardinales transfinitos junto al aportado por el método de la diagonal.

El infinito actual es imprescindible tanto para el desarrollo de la Matemática como para su enseñanza, ya que la acepción del infinito en acto permite establecer proposiciones y teoremas y además, favorece la validación, por ejemplo: en los textos clásicos de Cálculo y Análisis Matemático de Nivel Medio y Superior, el tema de límites generalmente se propone en dos etapas, la primera relacionada con el trabajo intuitivo del concepto, y la segunda etapa el trabajo formal; la definición del concepto utilizando el modelo $\varepsilon-\delta$. Consideramos que en la primera etapa cobra importancia el infinito potencial (visto como proceso reiterativo) y en la segunda etapa cobra importancia el infinito en acto (proceso infinito que culmina). De lo anterior consideramos que el profesor de matemáticas, debe conocer estas acepciones del infinito, manejar los dos tipos de infinito resultan fundamentales para el desarrollo en la escuela de temas de Cálculo y Análisis, como el de límites, integral, etc.

\subsection{Principio de Exhaución}

El Principio de Exhaución establece que: Si a una cierta magnitud A le quitamos una parte B no menor a la mitad de A, y si del resultado de esta operación otra vez quitamos una parte no menor a su mitad y así, una vez y otra vez, tendremos como resultado una cantidad menor que cualquier otra dada inicialmente.

El Principio de Exhaución puede reformularse, con matemática moderna, de la siguiente forma: Si $\epsilon$ y $A$ son ciertas cantidades positivas fijadas inicialmente y si restamos a $A$ la cantidad: $B=A-\frac{A}{2+h} \geq \frac{A}{2}, h \geq 0$ no menor a la mitad de $A$, entonces, tenemos que $A-B=A-\left(A-\frac{A}{2+h}\right)=\frac{A}{2+h}=x_{1}$. Y si nuevamente a: $A-B=x_{1}$ se le resta una cantidad no menor a su mitad se obtiene: $x_{1}-\left(x_{1}-\frac{x_{1}}{2+h}\right)=\frac{x_{1}}{2+h}=x_{2}$. Y ya que: $x_{1}=\frac{A}{2+h}$ tenemos, entonces: $x_{2}=\frac{A}{(2+h)^{2}}$. Luego, si a $x_{2}$ le restamos nuevamente una cantidad no menor a su mitad tenemos que: $x_{2}-\left(x_{2}-\frac{x_{2}}{2+h}\right)=\frac{A}{(2+h)^{3}}=x_{3}$. Y en general se tiene que: $x_{n}=\frac{A}{(2+h)^{n}}$ y de ahí que:

$$
\lim _{n \rightarrow \infty} x_{n}=\lim _{n \rightarrow \infty} \frac{A}{(2+h)^{n}}=0
$$


es decir, $x_{n}$ es menor que cualquier cantidad $\epsilon>0$ prefijada inicialmente. De esto se deduce que el Principio de Exhausción de Eudoxio es equivalente a afirmar que $\lim _{n \rightarrow \infty} \frac{1}{(2+h)^{n}}=0$, que es otra forma de enunciar el Principio de Arquimedes o Propiedad Arquimediana:

Las magnitudes de dos segmentos no pueden tener una razón si la magnitud menor no puede hacerse más grande que la mayor mediante una repetición determinada de veces.

Lo que a su vez equivale a decir que:

Si se dan dos segmentos de recta $\alpha$ y $\beta$ con longitudes distintas de cero y siendo $\alpha$ menor que $\beta$ entonces, repitiendo un número suficientemente grande de veces la menor llegaremos a superar siempre a la mayor.

En términos modernos podemos enunciarla así:

Propiedad Arquimediana: Si $\alpha$ y $\beta$ son dos números no negativos cualesquiera distintos de cero, tales que $\alpha<\beta$ entonces existe un número natural $n$ tal que: $n \alpha>\beta$.

Que se traduce simplemente en: ¡No hay distancia que no pueda superarse con el múltiplo de otra!

Ahora bien, ¿Cómo sabemos que: $\lim _{n \rightarrow \infty} \frac{1}{(2+h)^{n}}=0$ ? O dicho de otra forma, ¿Cómo sabemos que: $\left(\frac{1}{2+h}\right)^{n}<\epsilon$ a partir de una cierta $n_{0} \in \mathbb{N}=\{1,2,3, \ldots\}$ ? Para ver la veracidad de esto utilizaremos la desigualdad de Bernoulli, con ello se tiene que: $0<\left(\frac{1}{2+h}\right)^{n}<\frac{1}{2^{n-1} n h}<\frac{1}{n h}<\frac{1}{n} ; h>1$. Pero resulta que: $\lim _{n \rightarrow \infty} \frac{1}{n}=0$ y de ahí se concluye que: $\lim _{n \rightarrow \infty}\left(\frac{1}{2+h}\right)^{n}=0$, ya que se encuentra entre dos límites que tienden a 0 , es decir:

$$
0 \leq \lim _{n \rightarrow \infty}\left(\frac{1}{2+h}\right)^{n}<\lim _{n \rightarrow \infty} \frac{1}{n}=0
$$

Ahora, todo parece ir muy bien siempre y cuando podamos decir algo verdaderamente fuerte acerca de que: $\lim _{n \rightarrow \infty} \frac{1}{n}=0$, pero entonces caemos en otra situación: Si no aceptamos que $\lim _{n \rightarrow \infty} \frac{1}{n}=0$, lo que equivale a decir que $\frac{1}{n}<\epsilon$ a partir de una cierta $n_{0} \in \mathbb{N}$ y cualquier $\epsilon>0$ prefijado inicialmente, tendremos entonces que aceptar lo contrario, es decir que, $\frac{1}{n} \geq \epsilon, \forall n \in \mathbb{N}$, lo que implicaría que $n \epsilon \leq 1, \forall n \in \mathbb{N}$ lo que equivale a decir que no importa cuántas veces repitamos $\epsilon$ nunca podremos superar una cantidad distinta de 1, lo que está en franca contradicción con el Principio de Arquímedes, y de la intuición misma que nos indica que si $\epsilon=1$ tendríamos a los números naturales acotados por 1, es decir, que todos los números naturales son menores o iguales a 1 . Todo esto nos lleva a considerar que el Principio de Exhausción se basa en la Propiedad Arquimediana, y Eudoxio lo utilizó primero que Arquímedes. Bajo esta perspectiva se tiene entonces que un proceso infinito $P_{n}$ tiene situación límite siempre que una de sus variables de estado $v_{k}$ lleguen a ser menor que cualquier cantidad $\epsilon>0$ prefijada de antemano, por pequeña que ésta sea.

Según González (2007), en un proceso infinito pueden existir varias variables asociadas a él, estas constituyen su espacio fase, no obstante, muchas de esas variables pueden pertenecer a otro espacio fase; la clave de la cuestión es no confundir las variables de estado de distintos procesos infinitos.

En el caso de la curva escalonada tratada en el artículo, el proceso infinito nos lleva a considerar que, la variable $v_{1}=S=a+b$ su longitud siempre es constante, y mediante el Principio de Exhaución de Eudoxio podemos determinar que, en cada etapa no se sustrae una cantidad no menor a su mitad y por ello, la longitud $S$ no puede hacerse menor que cualquier cantidad $\epsilon>0$ prefijada de antemano, es decir, $S$ no satisface el Principio de Exahusción de Eudoxio. No obstante, eso no significa que la curva escalonada no constituya un proceso infinito, de hecho lo es, pero para determinar su situación límite debemos considerar otras variables asociadas a él, como las longitudes horizon- 
tales de los escalones, sólo así y mediante el Principio de Exhaución de Eudoxio podemos dar un sustento formal a la conexión entre el proceso infinito y la situación limite, si es que existe.

\subsection{Elementos teóricos y metodológicos}

La elaboración del diseño de exploración de situaciones que implican interpretación de procesos infinitos, se sustenta en los aportes de Duval, R. (1993). Según el investigador, los estudiantes empiezan a asimilar y aprender un concepto cuando lo identifican en sus distintas representaciones (registros): gráfico, numérico, algebraico, verbal y cuando los estudiantes, son capaces de cambiar de registro de representación, es decir, que el concepto en cuestión, puedan a través de una transformación llevarlo de una representación gráfica a una algebraica, etc. El diseño de exploración, contiene actividades relacionadas al concepto de límite finito, límite infinito, límite al infinito, en cual se pueden estudiar los procesos infinitos y dichas actividades están enmarcadas en distintos registros de representación. En este reporte sólo mostraremos los análisis de las actividades que están indicadas en el registro de representación gráfica-geométrica y en las cuáles se motiva a los estudiantes a la interpretación de los procesos infinitos.

Para esta investigación se trabajó con un grupo de 16 alumnos del segundo semestre de Licenciatura en Matemáticas, de la Universidad Autónoma de Guerrero, los cuales ya habían cursado la materia de Cálculo I y II. Se organizó al grupo en equipos, a cada miembro del equipo se le entregó el diseño, la instrucción que se dió fue que discutieran las actividades en equipos y contestaran el cuestionario, para posteriormente comentar las soluciones de manera grupal. Se utilizó equipo de audio para grabar las discusiones de los equipos, dichas actividades se trabajaron en una sóla sesión de 3 horas, donde el papel del profesor fue orientar y favorecer las discusiones sobre las actividades planteadas.

Cabe mencionar que las actividades propuestas para su análisis en este trabajo fueron elaboradas por los investigadores, a partir de revisar libros de texto clásicos de Cálculo y Análisis Matemático.

\subsection{Análisis de las interpretaciones}

A continuación se analizan las respuestas dadas por los estudiantes, cuando se enfrentan a procesos infinitos.

Actividad 1. Si consideramos un triángulo rectángulo como el que se muestra en la figura 2, y pensamos en dividir el cateto $a$ en un número $n$ creciente (al infinito) de partes iguales, y construimos la curva escalonada, entonces, la curva escalonada está cada vez más cerca de la hipotenusa. En la situación límite de este proceso infinito, ¿ocurre que la curva escalonada sea igual a la hipotenusa?

Comentarios previos al análisis de esta actividad:

- Debemos observar que la situación es mucho más compleja, en realidad se trata de una sucesión de curvas escalonadas, que pueden ser funciones al hacer una rotación $0<\theta<\frac{\pi}{2}$, cuyo límite tiende a la hipotenusa. Sin embargo, cada curva escalonada siempre mide $a+b$. Esto no debe ser una contradicción, ya que el hecho de que las curvas escalonadas converjan puntual y uniformemente a la hipotenusa, no tienen por qué coincidir con el valor de su longitud.

- Jungk, W. et al., (1985). Considera que en las primeras clases sobre el tema de límites, deben considerarse ejemplos en los cuales los alumnos puedan reconocer que: "El concepto de límite tiene que definirse exactamente con la utilización exclusiva de los conceptos matemáticos, para evitar toda contradicción." Es indiscutible lo que afirma el autor, sin embargo, consideramos importante la implementación de situaciones que permitan al estudiante favorecer su interpretación, en específico el relacionado con el límite. 


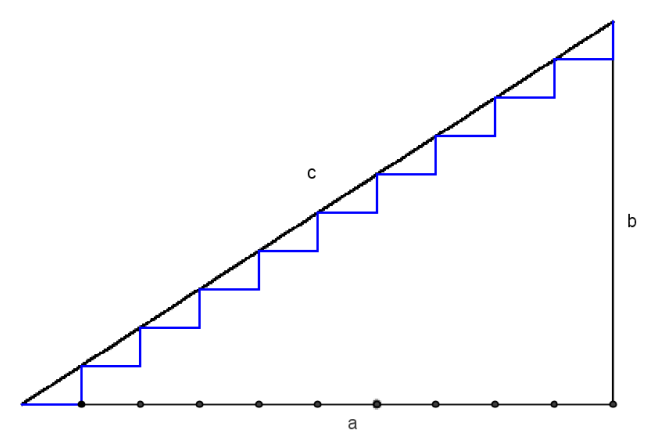

Figura 1.2

Algunas respuestas encontradas son:

a) No, porque aún cuando los escalones sean cada vez más pequeños siempre existirá un hueco entre ellos y no permitirá la igualdad entre la curva escalonada y la hipotenusa.

Observamos que en este caso, los estudiantes utilizan el infinito potencial como argumento para explicar que la curva escalonada no puede llegar a coincidir con la hipotenusa, pero no logran deducir, que la sucesión de las curvas escalonadas converge a la hipotenusa.

b) No ocurre eso, porque la curva escalonada sería igual a la hipotenusa si tendiera al infinito.

En este caso, los alumnos, aceptan que en la situación límite, la curva escalonada tiende a coincidir con a la longitud de la hipotenusa. Podemos observar que los alumnos utilizan la idea de infinito potencial en la explicación del proceso, y además cuando establecen que la curva escalonada tiende a la hipotenusa, suponen que el proceso tiende a terminar (el infinito en acto). Se observa en este caso. Que hay incluso un error conceptual, ya que el hecho de que las curvas escalonadas convergen a la hipotenusa, no quiere decir que las longitudes tienen que coincidir, pues son situaciones distintas. Resulta que la longitud de la curva escalonada siempre es constante, por lo que en la situación límite sigue teniendo tal longitud.

A continuación mostramos la transcripción de audio, de una entrevista a dos de las estudiantes que participan en la actividad:

Profesor: ¿Cuál es su opinión acerca de la situación límite de la curva escalonada, siempre que el proceso de división del cateto adyacente es infinito?

Leticia: Bueno, que llegado un momento en que el cateto se ha dividido por un número $n$ de veces, la curva escalonada se va cerrando, se va acercando a esta línea(señala la hipotenusa del triángulo dado)... Y la situación límite es cuando ya llegamos a un extremo. Es decir, la situación límite es el acercamiento a la hipotenusa, entonces la curva escalonada sólo se acerca (tiende) a la hipotenusa, pero no llega a coincidir, siempre habrá una diferencia.

Arlette: Veo que la curva escalonada se origina dependiendo de las divisiones del cateto adyacente, su longitud va a permanecer igual, pero lo que va a cambiar es las partecitas(triángulos o espacios)...se harán más chiquitos, si se siguen haciendo más chiquitos los espacios(triángulos), la longitud de la curva escalonada se acerca a la hipotenusa pero no llega a ser igual.

Actividad 2. Si consideramos un segmento de la curva de $f(x)$, como el que se muestra en la figura 3, y pensamos en dividir el intervalo $(b-a)$ en un número $n$ creciente (al infinito) de partes iguales, y construimos la curva escalonada, entonces, a pesar de que la curva escalonada está cada vez más cerca de la curva de $f(x)$, en 
la situación límite de este proceso infinito no ocurre que sea igual a la curva. ¿qué explicación le das a esta situación?

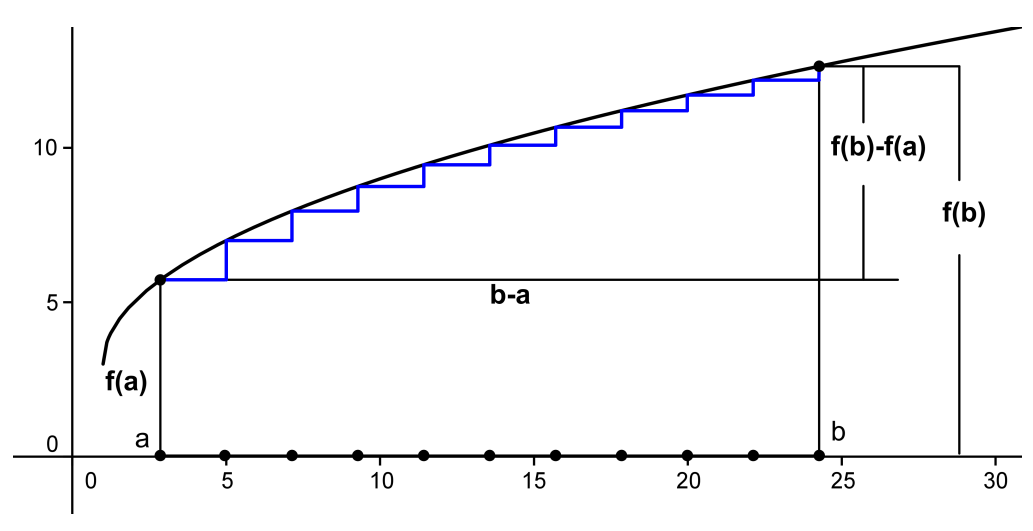

Figura 1.3

Algunas respuestas encontradas son:

c) La curva escalonada nunca coincidirá con la curva, porque aun cuando los escalones sean cada vez más chicos existirá un hueco entre ellos y no permitirá la igualdad entre la curva escalonada y la curva $f(x)$.

d) A pesar de que tengamos infinidades de divisiones del intervalo, nunca será igual a la curva.

e) En el proceso infinito no ocurre que la curva escalonada sea igual a la curva porque sólo se toma en cuenta un intervalo para dividirlo en $n$ partes iguales y como no se toma en cuenta la otra parte de la función eso hace que la curva escalonada no sea igual a la curva $f(x)$.

A continuación mostramos la transcripción de audio, de una entrevista a dos de las estudiantes que participan en la actividad:

Leticia: Aquí es más claro, más fácil ... porque es el mismo problema que tuvieron los griegos al inscribir polígonos regulares en un círculo, se origina un proceso infinito y en este caso el proceso tiende a culminar [Confunde la situación]. Digo que es más claro, porque aquí sí puedo llegar hacer coincidir la curva escalonada al segmento de curva, y no la curva escalonada a la recta (en el caso de la hipotenusa). Se llega hasta cierto punto pero no necesariamente tienen que coincidir, el límite es la curva ... algo que está acotado, por eso se empezó hablar del límite (La curva escalonada sólo tiende a la curva pero nunca es igual en longitud, siempre habrá una diferencia constante).

Arlette: La curva escalonada no llega a coincidir con el segmento de curva ... la longitud de la curva escalonada, sería $b-a+f(b)-f(a)$, si incremento el número de rectángulos, la longitud de la curva escalonada llegará a cambiar. Si hago más divisiones en b-a, incremento el número de los rectángulos, la suma de las áreas de los rectángulos llegan a agotar el área de la región, aja. Pero la curva escalonada nunca llega a coincidir, porque sigue habiendo espacios chiquitos, chiquitos, chiquitos, ...

Actividad 3. Si consideramos un segmento de la curva de $f(x)$, y pensamos en dividir el intervalo $b-a$ en un número $n$ creciente (al infinito) de partes iguales, y construimos los rectángulos bajo la curva como se muestra en la siguiente figura, entonces, a medida que el número de divisiones crece, la suma de las áreas de los rectángulos se aproxima más al área bajo la curva. Así en la situación límite de este proceso infinito, la suma de las áreas de los rectángulos es igual al área bajo la curva en dicho intervalo. ¿Cuál es la explicación que se puede dar en este caso?

Algunas respuestas encontradas: 


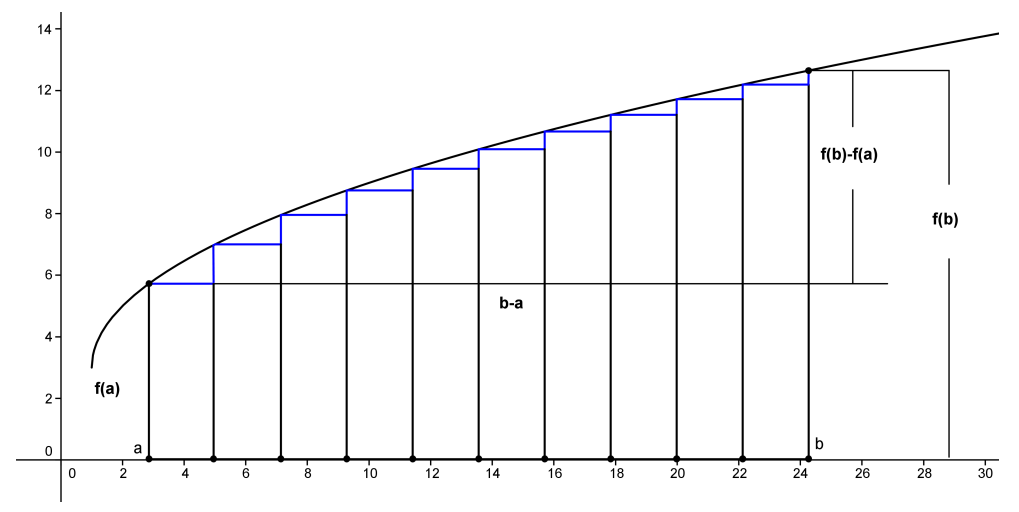

Figura 1.4

f) La suma de las áreas de los rectángulos llega a coincidir con el área bajo la curva, en la situación límite del proceso infinito, porque los triángulos que sobran al dibujar los rectángulos son cada vez más pequeños al aumentar el número de rectángulos en este intervalo.

En este caso observamos que, implícitamente los estudiantes utilizan la definición de integral definida como el límite de una suma de Riemann, y a diferencia de la situación anterior, al parecer, en este caso les es más fácil concluir que el proceso tiende a terminar, y aunque no lo explicitan, aceptan que la curva escalonada llega a coincidir con la curva definida en el intervalo $[a, b]$. Sin embargo, igual que en los casos anteriores, la sucesión de curvas escalonadas tienen longitud constante, y aunque convergen al segmento de curva, no necesariamente coinciden sus longitudes, la pregunta natural que surge es ¿qué condiciones permiten establecer la igualdad?, la teoría de la medida posibilita tal explicación, sin embargo en este trabajo no nos proponemos tal discusión.

Otras respuestas encontradas:

g) El límite de la suma de áreas es igual al límite de la suma de Riemann, es decir, que es la suma de las áreas bajo la curva de los rectángulos circunscritos e inscritos, y la suma del área bajo la curva es un número que se encuentra entre estos dos valores.

h) A medida que la división del intervalo tiende a ser infinito cada vez más se cubre más área de la región bajo la curva, ya que los espacios entre la curva y los rectángulos es cada vez menor, por ello, cuando tiende a infinito el número de divisiones del intervalo, el área de los rectángulos es igual al área de la región bajo la curva.

Según las respuetas anteriores, observamos que en los estudiantes prevalece la idea de infinito potencial, pues argumentan que la curva escalonada nunca llega a coincidir con el segmento de curva de $f(x)$, sin embargo, cuando reflexionan sobre el área, ellos dicen que en el proceso infinito la suma de las áreas de los rectángulos coincide con el área bajo la curva en el intevalo dado.

A continuación mostramos la transcripción de audio, de una entrevista a dos de las estudiantes que participan en la actividad:

Leticia: En el infinito la suma de las áreas de los rectángulos es igual al área de la región, no siempre habrá una diferencia, se puede hacer una aproximación de afuera hacia dentro. Si se construyen dos fórmulas y se les determina el limite qué te va a dar ... la curva. Si se incrementa el número de rectángulos, la suma de las áreas de los rectángulos serán una aproximación...nunca llegarán a coincidir. Qué concluyen los griegos, encontraron el valor de $\pi$... para hacerlo, empezaron con el cuadrado, y luego incrementaron el número de lados a los polígonos, ...cómo se convencieron los griegos sobre la aproximación a $\pi$. Se acercaron por dos lados, no importa qué tanto se incrementen los lados de los polígonos siempre 
sería así,... es como cuando empiezan a estudiar el límite a partir de sucesiones, a poder determinar que cierta parte se puede dividir y hacerla más pequeña haciendo un proceso infinito, pero que llega a un límite, el acotamiento siempre fue un círculo, la situación límite es el círculo (el límite de un proceso infinito).

\subsection{Conclusiones generales}

De la actividad 1.

- De las respuestas de los cuestionarios, y de las transcripciones mostradas, observamos que en los estudiantes prevalece la idea del infinito potencial cuando interpretan procesos infinitos, en el caso de la sucesión de curvas escalonadas, la mayoría considera que estas tienden a la hipotenusa, y sin embargo siempre habrá una diferencia infinitésima. Por otro lado, al mencionar que las curvas escalonadas no sobrepasan a la hipotenusa, está el hecho implícito que se supone la existencia del infinito en acto.

De la actividad 2.

- Se trata de una función monótona, por lo que la sucesión de curvas escalonadas tiene longitud constante, pero la situación límite de la sucesión de curvas escalonadas es el segmento de curva. Es decir, el proceso infinito (la sucesión de curvas escalonadas) tiene una situación límite (el segmento de curva).

De manera análoga a la actividad 1, no debe confundirse la longitud de cada curva escalonada con la longitud del semento de curva. Quizá sea necesario discutir el comportamiento de la curva poligonal, yaque parece más natural que tal curva poligonal tienda a coincidir en la situación límite con el segmento de curva. Lo anterior se posibilita, a partir de utilizar herramientas del Cálculo o del Análisis Matemático, en específico las sumas de Darboux (Valdés, 1983).

- La interpretación que hace la mayoría de los estudiantes, está relacionada al hecho de que el caso es más sencillo, ya que recuerdan que el límite de la sumas de las áreas de los $n$ rectángulos tiende al área bajo la curva, y la mayoría concluye que en este caso la curva escalonada coincide con el segmento de curva. Observamos que incluso hay un error conceptual, ya que el hecho de que el límite de las curvas escalonadas tiende al segmento de curva, no significa que la longitud de cada curva escalonada coincida con la longitud del segmento de curva, de hecho puede o no coincidir. Se puede probar que la longitud de la curva poligonal es la que tiende a coincidir al segmento de curva y no la longitud de las curvas escalonadas a la longitud del segmento de curva.

- Una sola estudiante sostiene que las curvas escalonadas tienden al segmento de curva, sin que necesariamente coincidan, en este y en el caso anterior los alumnos consideran que el proceso infinito tiende a culminar.

De la actividad 3.

- Es verdad que el límite de la suma de las áreas de $n$ rectángulos tiende al área bajo la curva, en este caso se tienen herramientas matemáticas para este tipo de argumentación, las sumas inferiores y superiores, cuyo límite puede tender al área bajo la curva. En este caso la curva poligonal tiende al segmento de curva, y el límite de la sucesión de curvas escalonadas converge al segmento de curva, sin que necesariamente coincidan sus longitudes.

- Los estudiantes establecieron que el proceso infinito en este caso, tiende a terminar, pero no distinguieron el comportamiento de la curva poligonal, y la sucesión de las curvas escalonadas. Si embargo, notamos que la idea del infinito potencial es la más usual cuando argumentan sobre la interpretación de estos procesos infinitos.

De acuerdo a las anteriores situaciones y de la revisión de algunos textos clásicos de Cálculo y Análisis, podemos establecer que el tipo de infinito que es usado más en la escuela, es el infinito potencial. Consideramos que esto no es casual, ya que en los textos se identifica que cuando introducen el concepto de límite, generalmente aparecen 
como ejemplos situaciones de tipo geométrico o numérico que involucran el concepto de infinito y sus acepciones: potencial y actual. Por otra parte, en los argumentos que dan los estudiantes, notamos que hacen uso del infinito potencial para explicar en este caso, que la sucesión de curvas escalonadas tienden a la hipotenusa. Sin embargo, habría que decir, que desde nuestra posición, el infinito potencial presupone la existencia del infinito en acto.

Por otro lado, al analizar las interpretaciones sobre procesos infinitos, se observa incluso que los estudiantes, confunden el hecho de que la curva escalonada tiende a la curva principal, definida en el intervalo $[a, b]$, la confusión se da a partir, de que no identifican que en la situación límite la sucesión de curvas escalonadas tiende a la hipotenusa, sin que sus longitudes coincidan.

Desde el punto de vista didáctico, las situaciones analizadas, pueden permitir generar una discusión formal sobre cómo determinar determinar la longitud de una curva, ello permitirá, de paso reflexionar sobre la longitud de las curvas escalonadas, la longitud de la curva poligonal y su relación con la longitud de la curva en el intervalo dado.

Desde nuestra posición, el objetivo propuesto en el artículo se ha logrado, al mostrar el tipo de infinito que utilizan los estudiantes cuando se enfrentan a procesos infinitos. Éstas interpretaciones las consideramos fundamentales, porque pueden ser el punto de partida para investigaciones futuras en este campo.

\subsection{Recomendaciones}

1. Tener presente que no todos los procesos infinitos tienen situación límite. Lo anterior significa que la situación límite es un ente independiente, es decir, la situación límite no deviene de un proceso infinito pero sí podemos asociarle un proceso infinito, y sólo bajo esa condición es que dicho proceso tiende a culminar, en otras palabras tiene límite.

2. Tomar en cuenta que para que una situación límite se le pueda asociar un proceso infinito $P_{n}$, basta con que una de sus variables $v_{k}$ del proceso infinito, cumpla con el principio de exhausción, de esa manera se garatizará que los valores de la variable $v_{k}$ lleguen a ser menor que cualquier cantidad $\epsilon>0$ prefijada de antemano, por pequeña que ésta sea.

3. Considerar la relación entre el principio de Exhaución y la Propiedad Arquimediana, que resultan fundamentales para la explicación de la situación límite de los procesos infinitos.

\section{Bibliografía}

[1] Antibí, A. (1996). Didáctica de la Matemática.Métodos de resolución de problemas: Límites. México: Universidad Autónoma de Guerrero.

[2] Camacho, A. y Aguirre, M. (2001). Situación didáctica del concepto de límite infinito. Análisis preliminar. Revista Latinoamericana de Investigación en Matemática Educativa, 4(3), 237-265.

[3] Cornu, B. (1983). Apprentissage de notion de límite: conceptions et obstacles. (These de doctorat). Universitè de Grenoble.

[4] De Lorenzo, J. (2001). El infinito matemático. Revista de Divulgación Matemática (pp. 4-9).

[5] Díaz, M. (2009). El Método de Exhaución . [Versión Electrónica], Revista Alternativa, 19, 1-25.

[6] Duval, R. (1993). Semiosis y Noesis. En Lecturas en Didáctica de la Matemática: Escuela Francesa. México: Sección de Matemática Educativa del CINVESTAV-IPN.

[7] Engler, A. et al.(2008). El límite infinito: Una situación didáctica. Revista Premisa, 10/36, 11-21.

[8] González, J. (2007). Geometría una reflexión infinita: Un análisis de las posibilidades y alcances de los conceptos de la Geometría. Universidad Autónoma de Guerrero: México. 
[9] Hernández, F. (2003). Teoría de Conjuntos: Una Introducción (Segunda Edición). México: Sociedad Matemática Mexicana.

[10] Hitt, F. (2003). El concepto de infinito: obstáculo en el aprendizaje de límite y continuidad de funciones. En E. Filloy (Ed.), Matemática Educativa: Aspectos de la Investigación Actual (pp. 91-111). México: Centro de Investigación y de Estudios Avanzados - FCE.

[11] Jungk, W. et al.,(1982). Conferencias sobre Metodología de la Enseñanza de la Matemática 3. Cuba: Pueblo y Educación.

[12] Le Goff, J-P. (2001). La perspectiva y el infinito geométrico. Revista de Divulgación Matemática (pp. 30-35).

[13] Moreno, L. y Waldeg, G. (1986). Cálculo Avanzado: Introducción al Análisis (1a. Parte). México: Sección Matemática Educativa del IPN.

[14] Reyes, L. E. (2012). Identificación y caracterización de dificultades entorno a la noción de límite en el infinito en estudiantes de Nivel Superior.Tesis no publicada. Universidad Autónoma de Guerrero: México.

[15] Valdés, C. (1983). Análisis Matemático, Tomo II. Cuba: Pueblo y Educación. 